\title{
Automatic beam alignment in the Garching 30-m prototype of a laser-interferometric gravitational wave detector
}

\author{
G. Heinzel ${ }^{1}$, A. Rüdiger, R. Schilling, K. Strain ${ }^{2}$, W. Winkler, J. Mizuno ${ }^{3}$, \\ K. Danzmann ${ }^{4}$ \\ Max-Planck-Institut für Quantenoptik, D-85748 Garching, Germany
}

Received 28 August 1998; accepted 2 December 1998

\begin{abstract}
We describe a system for complete autoalignment of a suspended, power-recycled Michelson interferometer. All ten angular degrees of freedom are controlled by servo systems, thus ensuring optimal interference and fixing all beams in space. The methods and results are applicable to laser-interferometric gravitational wave detectors, and possibly also to other types of sensitive interferometers. (C) 1999 Elsevier Science B.V. All rights reserved.
\end{abstract}

PACS: 04.80.Nn; 07.60.Ly; 42.25.Hz; 95.55.Ym

Keywords: Michelson interferometer; Automatic beam alignment; External modulation; Schnupp modulation; Differential wavefront sensing; Power recycling

\section{Introduction}

Several large laser interferometers are currently under construction [1-4], with the aim of detecting gravitational waves from astrophysical sources. These instruments are basically long-baseline Michelson interferometers with the main components (beamsplitter and mirrors) suspended as pendulums in vacuum. A gravitational wave of the proper characteristics will induce a phase shift of opposite sign on the light in the two (orthogonal) arms, causing a tiny offset from the nominal operating point. The resulting light amplitude at the detector's output port is detected with one of several possible modulation schemes (see e.g. Refs. [5,6] and references in Ref. [6]).

The shot-noise limited sensitivity of such a detector improves with the light power in the arms. All planned detectors will employ power recycling (PR) in order to increase this light power [7,8]. The Michelson operating at the dark fringe point (interference minimum at the output port) reflects almost all light back towards the laser. By placing a mirror $\left(\mathrm{M}_{\mathrm{PR}}\right.$ in Fig. 1) between laser and beamsplitter, a kind of Fabry-Perot cavity (the 'PR cavity') is formed, in which the light power can be resonantly enhanced. Most, but not all, detectors additionally plan to use a Fabry-Perot cavity in each of the two arms. This is not considered in this paper. The methods and results presented here should however also be useful for these cases.

To make the interferometer work optimally all optical components need to be well aligned, and to remain so for extended periods of time. 'Alignment' in this paper refers to angular alignment of mirrors and beamsplitter, assuming the relevant longitudinal loops to be working, in particular the PR cavity to be resonant in its fundamental mode. In the following we

\footnotetext{
${ }^{1}$ E-mail: ghh@mpq.mpg.de

${ }^{2}$ University of Glasgow, Department of Physics and Astronomy, Glasgow G12 8QQ, UK.

${ }^{3}$ Now at: The Institute of Physical and Chemical Research (RIKEN), Optical Engineering Laboratory, Hirosawa 2-1, Wako, Saitama 351-0198, Japan.

${ }^{4}$ Also at: Universität Hannover, Institut für Atom- und Molekülphysik, Appelstrasse 2, D-30167 Hannover, Germany.
} 


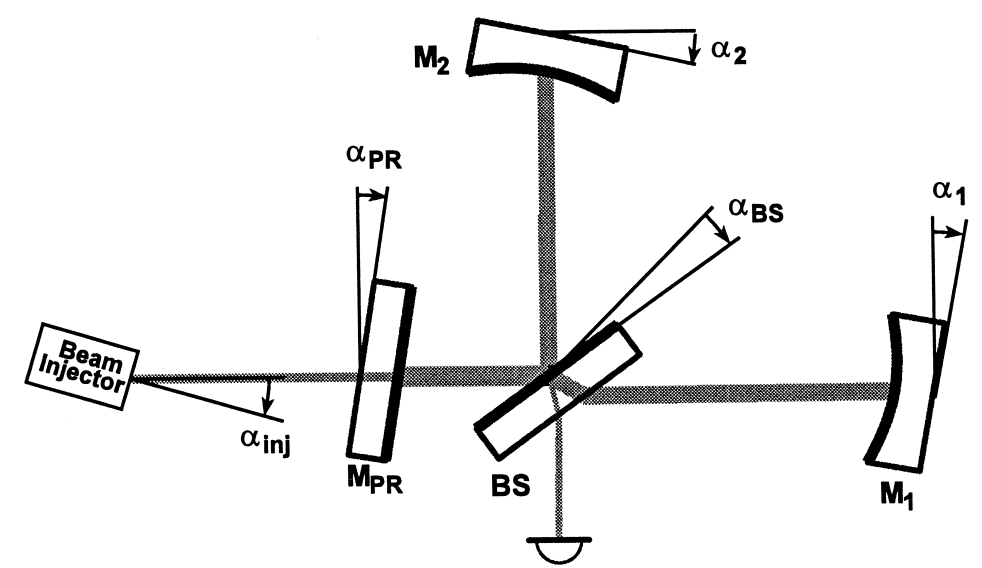

Fig. 1. Schematic diagram of a power-recycled Michelson interferometer showing all possible angular misalignments $\alpha$ in one dimension. Beams are shown in their nominal positions, whereas the components are shown misaligned by exaggerated angles.

consider only a single angular dimension $\alpha$ for each component, the orthogonal direction to be handled equivalently. In this paper, we count an angle as positive if it is rotated clockwise from a reference direction. Fig. 1 schematically shows all possible misalignments in one dimension.

The required performance can only be achieved with an automatic alignment system. We use and extend here the differential wavefront sensing technique $[9,10]$, which allows to detect misalignments of two interfering beams against each other. Referring to Fig. 1, we can separately consider the following alignment tasks:

Michelson alignment. For optimal interference contrast, the axes of the beams returning from the two arms need to coincide when they recombine at the beamsplitter. The two degrees of freedom to be controlled are (i) the 'differential' term $\alpha_{\text {diff }}=\alpha_{1}+\alpha_{2}$ of the end-mirror alignments, and (ii) its equivalent in the other dimension. Good alignment of the Michelson interferometer is also necessary to suppress the coupling of beam jitter into noise in the output signal [11].

Power recycling cavity alignment. If we assume the incoming laser beam to be fixed in space, the PR cavity's axis needs to coincide with the incoming beam's axis in order to obtain optimal coupling of the laser light into the PR cavity. There are four degrees of freedom to be controlled: (i) the PR mirror's alignment $\alpha_{\mathrm{PR}}$, (ii) the common mode term $\alpha_{\text {comm }}=\alpha_{1}-\alpha_{2}$ of the end mirror alignments, and (iii,iv) their equivalents in the other dimension.

Spot positions on mirrors. If the above two systems are working, there are two more uncontrolled optical components: The beam injector ${ }^{5}$ (which is usually also suspended as a pendulum), and the beamsplitter. These two components define the position of the beam spot on the far mirrors $\mathbf{M}_{1}$ and $\mathbf{M}_{2}$, respectively. Although these spot positions in principle do not affect the operation of the interferometer, in practice one also needs to control these four degrees of freedom for various reasons, such as wandering spot positions on mirrors and photodetectors, scattered light variations, inhomogeneities of mirror coatings, etc.

Thus there are $5 \times 2$ degrees of freedom in a power-recycled Michelson interferometer (beam injector, PR mirror, beamsplitter and two end mirrors) to be controlled in order to fix all beams in space. The beam positions are then completely defined by the lateral positions of the beam injector and of the sensors that determine the beam spot positions on the end mirrors.

The outline of this paper is as follows: In Section 2 we present the theory necessary to obtain the alignment error signals. The general experimental set-up is described in Section 3, followed by a description of design and performance of the alignment loops in Section 4.

\section{Theory of alignment detection}

Hefetz et al. [12] developed a general formalism to describe arbitrary misaligned fields using operators in modal space. Here, however, we concentrate on the practical computation of alignment error signals. Our analysis is restricted to the two

\footnotetext{
${ }^{5}$ By 'beam injector' we mean the last component determining the position and angle of the beam hitting the PR mirror. In our prototype this is a fiber output coupler suspended as a pendulum, whereas in the large interferometers it will probably be a suspended beam-steering mirror.
} 
lowest modes and emphasizes experimental aspects such as the geometry of the interferometer, various possible modulation schemes for a power-recycled Michelson interferometer, and the manipulation of the beam with lens systems to separate the error signals.

\subsection{Misalignments in the mode picture}

We assume a beam propagating along the z-axis, and again consider only one orthogonal direction $x$. It is well-known $[11,13,9]$ that small lateral or angular misalignments of a Gaussian $\mathrm{TEM}_{00}$ beam can conveniently be described by adding a small component of the first order mode $u_{1}$ to the dominating fundamental mode $u_{0}$. We will use the notation

$$
\begin{aligned}
& u_{0}(x, z)=C \exp \left(-\frac{x^{2}}{w^{2}(z)}\right), \\
& u_{1}(x, z)=C \frac{2 x}{w(z)} \exp \left(-\frac{x^{2}}{w^{2}(z)}\right),
\end{aligned}
$$

with

$$
C=\left[2 /\left(\pi w^{2}(z)\right)\right]^{1 / 4}
$$

Here $w_{0}=\sqrt{\lambda z_{\mathrm{R}} / \pi}$ is the beam radius at the waist, $w(z)=w_{0} \sqrt{1+\left(z / z_{\mathrm{R}}\right)^{2}}$ the beam radius elsewhere, $z_{\mathrm{R}}$ the Rayleigh range of the beam and $\lambda$ the wavelength. Note that the functions $u_{0}$ and $u_{1}$ do not contain the wavefront curvature (with radius of curvature $R(z)=z+z_{\mathrm{R}}^{2} / z$ ). The effects of the curvature are, however, taken into account in the formalism below. We will use a $\mathrm{TEM}_{00}$ beam described by $u_{0}$ as reference and compare a slightly misaligned $\mathrm{TEM}_{00}$ beam against this reference. We assume the misaligned beam to have the same waist size and longitudinal waist position as the reference.

At the beam waist $(z=0)$, the $u_{1}$ component is in phase with $u_{0}$ for lateral displacements $\Delta x$, and in quadrature for angular misalignments $\beta$ between the beams' axes:

$$
\begin{aligned}
& u_{0}(x-\Delta x, 0) \approx u_{0}(x, 0)+\frac{1}{w_{0}} \Delta x u_{1}(x, 0), \\
& u_{0}(x, 0) \exp \left(\mathrm{i} \frac{2 \pi}{\lambda} \beta x\right) \approx u_{0}(x, 0)+\mathrm{i} \frac{\pi w_{0}}{\lambda} \beta u_{1}(x, 0) .
\end{aligned}
$$

We will need to consider such a beam after propagation away from the waist $(z \neq 0)$. It can be shown that the above approximations can consistently be generalized as follows. A real (in-phase) $u_{1}$ component corresponds to a lateral separation of the beams' centers with the scaling factor $w(z)$, whereas an imaginary component corresponds to an angle $\gamma$ between the wavefronts with a scaling factor of $\lambda /[\pi w(z)]$. In the case of a tilt of the beams' axes by the angle $\beta$ (about the beam waist as pivot), that angle is given by $\gamma(z)=\beta z_{\mathrm{R}}^{2} /\left(z^{2}+z_{\mathrm{R}}^{2}\right)$. We thus have:

$$
\begin{aligned}
& u_{0}(x-\Delta x, z) \approx u_{0}(x, z)+\frac{1}{w(z)} \Delta x u_{1}(x, z), \\
& u_{0}(x, z) \exp \left(\mathrm{i} \frac{2 \pi}{\lambda} \gamma x\right) \approx u_{0}(x, z)+\mathrm{i} \frac{\pi w(z)}{\lambda} \gamma u_{1}(x, z) .
\end{aligned}
$$

These equations are valid for small misalignments, i.e. $\Delta x \lesssim w(z), \gamma \lesssim \lambda /[\pi w(z)]$.

In general we will have to consider combinations of lateral displacements and angular misalignments, i.e. beams given by (at their waist):

$$
u_{0}(x, 0)+\kappa_{1} u_{1}(x, 0)+\kappa_{2} \text { i } u_{1}(x, 0)=u_{0}(x, 0)+\kappa \exp \left(\mathrm{i} \theta^{\mathrm{w}}\right) u_{1}(x, 0) .
$$

We describe the 'character' of the misalignment at the waist by the angle $\theta^{\mathrm{w}}=\arctan \left(\kappa_{2} / \kappa_{1}\right)$, and its 'amount' by $\kappa$ $=\sqrt{\kappa_{1}^{2}+\kappa_{2}^{2}}$.

When a misaligned beam propagates, the first order mode $u_{1}$ acquires an additional phase shift with respect to $u_{0}$ which is given by the Guoy phase $\eta(z)=\arctan \left(z / z_{\mathrm{R}}\right)$ and which alters the ratio of lateral to angular misalignment. At a distance $z$ from the waist we then have

$$
u_{0}(x, z)+\kappa \exp \left\{\mathrm{i}\left[\theta^{\mathrm{w}}+\eta(z)\right]\right\} u_{1}(x, z) .
$$


For example, if we start with a beam that has a pure lateral displacement $\Delta x$ at its waist $\left(\theta^{\mathrm{w}}=0\right)$ and consider its propagation away from the waist, we find that the real part of the coefficient of $u_{1}$ decreases. If, however, we take into account the scaling factor $w(z)$, the physical displacement $\Delta x$ remains constant. At the same time an imaginary part of the coefficient of $u_{1}$ evolves, which corresponds to an angle $\gamma$ between the wavefronts caused by the now finite wavefront curvature.

In the following we will have to consider propagation through a system of lenses and segments of free space, characterized by its overall phase shift $\psi$. After the sytem we then have $\theta^{\mathrm{d}}=\theta^{\mathrm{w}}+\psi$ describing the character of the misalignment. $\theta^{\mathrm{d}}=0$ corresponds to a displacement between the beam's centers with parallel wavefronts, whereas $\theta^{\mathrm{d}}=90^{\circ}$ describes coinciding centers with an angle between the wavefronts. It will turn out that in the differential wavefront sensing scheme the detectors are only sensitive to that angle, such that $\theta^{\mathrm{d}}=90^{\circ}$ yields the maximum signal.

\subsection{Detection of misalignments}

The alignment of an interferometer is determined by the superposition of the axes of two or more beams. In the differential wavefront sensing scheme, one of these must be phase modulated (usually at a frequency of several MHz). The intensity of the interference pattern will then in general contain a term at the modulation frequency. The integral of this term over the whole cross section contains information about the longitudinal phase relationship of the two interfering beams and is usually exploited for the corresponding longitudinal loop. If the two beams' axes are not perfectly superimposed, the RF term in the intensity of the interference pattern will have a spatial structure that we exploit to obtain error signals containing information about misalignments of the interferometer.

Fabry-Perot cavity. In the case of a Fabry-Perot cavity (such as the PR cavity), the Pound-Drever-Hall method [14] is used for longitudinal locking. The directly reflected incoming beam (phase modulated with modulation index $m$ at a frequency $\omega_{\mathrm{m}}$ ) is taken as geometrical reference and can be written as

$$
a_{1}=c_{1} u_{0} \exp (\mathrm{i} \varphi)\left[J_{0}(m)+2 \mathrm{i} J_{1}(m) \cos \left(\omega_{\mathrm{m}} t\right)\right] \text {. }
$$

The beam leaking out of the cavity, assumed misaligned against this reference, is given by

$$
a_{2}=-c_{2}\left[u_{0}+\kappa \exp \left(\mathrm{i} \theta^{\mathrm{d}}\right) u_{1}\right]
$$

where $c_{1}$ and $c_{2}$ are positive constants, $\kappa$ and $\theta^{\mathrm{d}}$ represent the amount and character of the misalignment (see Eq. (8) and Section 2.3) and $\varphi$ is the phase difference between the fundamental mode terms of these two beams, arranged such that $\varphi=0$ for the resonance of the cavity. Although only the first two Bessel functions $J_{0}$ and $J_{1}$ appear in the equations, the following results can be shown to be valid for arbitrary modulation indices (assuming sinusoidal modulation and none of the modulation sidebands being resonant in the cavity). We compute the light intensity $\left|a_{1}+a_{2}\right|^{2}$ and determine the coefficients $I_{\omega_{\mathrm{m}}}$ of the terms that oscillate with $\omega_{\mathrm{m}} t$. The longitudinal error signal $V_{\mathrm{FP}}$ is found by integrating this $I_{\omega_{\mathrm{m}}}$ term over the whole cross section of the beam:

$$
V_{\mathrm{FP}}=\int_{-\infty}^{\infty} I_{\omega_{\mathrm{m}}}(x) \mathrm{d} x=4 c_{1} c_{2} J_{1}(m) \sin \varphi
$$

On the other hand, the alignment error signal $W_{\mathrm{FP}}$ is obtained with a split photodiode as the difference of the contributions from the two halves:

$$
W_{\mathrm{FP}}=\int_{0}^{\infty} I_{\omega_{\mathrm{m}}}(x) \mathrm{d} x-\int_{-\infty}^{0} I_{\omega_{\mathrm{m}}}(x) \mathrm{d} x=4 c_{1} c_{2} J_{1}(m) \sqrt{2 / \pi} \kappa \sin \left(\varphi-\theta^{\mathrm{d}}\right) .
$$

If the Pound-Drever loop is locked, we have $\varphi \approx 0$ and

$$
W_{\mathrm{FP}}=-4 c_{1} c_{2} J_{1}(m) \sqrt{2 / \pi} \kappa \sin \theta^{\mathrm{d}} .
$$

Looking back at Eqs. (6)-(8) we see that we get a signal for an angular misalignment between the wavefronts $\left(\theta^{\mathrm{d}}=90^{\circ}\right)$, but not for a lateral displacement.

In the above calculations we have assumed that the beam hits the center of the photodiode. In Ref. [9] it is shown that any offset between the centers of the interference pattern and the photodiode causes a reduction of the signal and spurious signals from higher-order modes, such as second order modes corresponding to a mismatch in beam size or curvature. For this reason it is necessary to keep the beam always centered on the quadrant diode (see Section 3.3).

Michelson interferometer. For the Michelson interferometer, again a modulation technique is employed to read out the Michelson phase near a dark fringe. The planned large-scale interferometers will use either Schnupp modulation [5] or external modulation [6]. We now treat these two cases, which have both been successfully used with autoalignment in the 30-m prototype at Garching. 
In Schnupp modulation (also called pre-modulation or frontal modulation), the incoming laser beam is phase modulated with modulation index $m$ at an angular frequency $\omega_{\mathrm{m}}$. An intentional length difference $\Delta l$ between the arms causes a fraction of the modulation sidebands to appear at the dark fringe port, where they act as local oscillator to detect the signal (and misalignment). We can write the amplitudes of the two beams from the arms as

$$
\begin{aligned}
& a_{1}=c_{1} \exp (\mathrm{i} \varphi / 2)\left[u_{0}+\frac{\kappa}{2} \exp \left(\mathrm{i} \theta^{\mathrm{d}}\right) u_{1}\right]\left[J_{0}(m)+2 \mathrm{i} J_{1}(m) \cos \left(\omega_{\mathrm{m}} t+\varepsilon\right)\right], \\
& a_{2}=-c_{2} \exp (-\mathrm{i} \varphi / 2)\left[u_{0}-\frac{\kappa}{2} \exp \left(\mathrm{i} \theta^{\mathrm{d}}\right) u_{1}\right]\left[J_{0}(m)+2 \mathrm{i} J_{1}(m) \cos \left(\omega_{\mathrm{m}} t-\varepsilon\right)\right] .
\end{aligned}
$$

Here $c_{1} \approx c_{2}, \varphi$ is the deviation of the Michelson phase from the dark fringe, and $2 \varepsilon=2 \Delta l \omega_{\mathrm{m}} / c$ is the phase difference of the modulation after one roundtrip in the arms.

With the same method as above we obtain as the signals for longitudinal locking (of the Michelson to a dark fringe) and alignment

$$
\begin{aligned}
& V_{\mathrm{MI}}=-8 c_{1} c_{2} J_{0}(m) J_{1}(m)\left[1-\left(\frac{\kappa}{2}\right)^{2}\right] \sin \varepsilon \sin \varphi, \\
& W_{\mathrm{MI}}=-8 c_{1} c_{2} J_{0}(m) J_{1}(m) \sqrt{2 / \pi} \kappa \sin \varepsilon \cos \varphi \sin \theta^{\mathrm{d}} .
\end{aligned}
$$

The longitudinal loop (dark fringe lock) uses $V_{\mathrm{MI}}$ as error signal and keeps that error signal very close to zero, thus enforcing the dark fringe condition $\varphi=0$. Again an alignment signal is obtained only from the angular misalignment between the wavefronts.

Another possible scheme to read out the phase of the Michelson interferometer is external modulation where in an additional Mach-Zehnder interferometer the beam leaving the interferometer through the detection port is brought to interference with a local oscillator beam, phase-modulated at $\omega_{\mathrm{m}}$, which is usually taken from one arm. The first autoalignment experiments in our prototype have used external modulation, which was later replaced by Schnupp modulation.

We now have to consider three interfering beams. We call their amplitudes $a_{1}$ and $a_{2}$ for the beams from the first and second arm, and $a_{3}$ for the local oscillator beam. According to the experimental setup described in Section 3 below, we assume $a_{3}$ to be taken from $a_{1}$ and thus perfectly aligned with it, whereas $a_{2}$ is misaligned against this reference. We can then write down the amplitudes

$$
\begin{aligned}
& a_{1}=c_{1} u_{0}, \\
& a_{2}=-c_{2}\left[u_{0}+\kappa \exp \left(\mathrm{i} \theta^{\mathrm{d}}\right) u_{1}\right] \exp (\mathrm{i} \varphi), \\
& a_{3}=c_{3} u_{0}\left[J_{0}(m)+2 \mathrm{i} J_{1}(m) \cos \left(\omega_{\mathrm{m}} t\right)\right] \exp \left(\mathrm{i} \Phi_{\mathrm{MZ}}\right) .
\end{aligned}
$$

Here $\varphi$ represents the deviation of the Michelson phase from the dark fringe, $\Phi_{\mathrm{Mz}}$ the phase of the Mach-Zehnder interferometer and $c_{1}, c_{2}$ and $c_{3}$ are positive constants with $c_{1} \approx c_{2}$ and $c_{3} \ll c_{1}$. Both $\varphi$ and $\Phi_{\mathrm{MZ}}$ are defined such that their nominal values at the operating point are zero. The signals for longitudinal locking (of the Michelson to a dark fringe) and alignment are found to be

$$
\begin{aligned}
& V_{\mathrm{MI}}=-4 c_{3} J_{1}(m)\left[c_{2} \sin \left(\varphi-\Phi_{\mathrm{MZ}}\right)+c_{1} \sin \Phi_{\mathrm{MZ}}\right], \\
& W_{\mathrm{MI}}=-4 c_{2} c_{3} J_{1}(m) \sqrt{2 / \pi} \kappa \sin \left(\varphi-\Phi_{\mathrm{MZ}}+\theta^{\mathrm{d}}\right) .
\end{aligned}
$$

At the proper operating point of the Mach-Zehnder, $\Phi_{\mathrm{MZ}}=0$, we have

$$
\begin{aligned}
& V_{\mathrm{MI}}=-4 c_{2} c_{3} J_{1}(m) \sin \varphi, \\
& W_{\mathrm{MI}}=-4 c_{2} c_{3} J_{1}(m) \sqrt{2 / \pi} \kappa \sin \left(\varphi+\theta^{\mathrm{d}}\right) .
\end{aligned}
$$

As before, an alignment error signal at the dark fringe operating point $(\varphi=0)$ is obtained only for an angular error, a tilt of the wavefronts at the detector (for $\theta^{\mathrm{d}}=90^{\circ}$ ).

Mach-Zehnder alignment. With external modulation, there is one more interferometer that needs to be aligned: the Mach-Zehnder interferometer. Using a recombination plate (RP in Fig. 4) that has the same dimensions and is parallel to the beamsplitter [15], the sensitivity of the interferometer's performance on the Mach-Zehnder alignment is already reduced. We now show how to obtain error signals that detect misalignments of the Mach-Zehnder. 
We start with the same amplitudes as in Eqs. (19)-(21) above, but now assume the Michelson to be perfectly aligned and instead the local oscillator beam $a_{3}$ to be misaligned. For simplicity we now also assume $c_{1}=c_{2}$. This leads to the light amplitudes

$$
\begin{aligned}
& a_{1}=c_{1} u_{0}, \\
& a_{2}=-c_{1} u_{0} \exp (\mathrm{i} \varphi), \\
& a_{3}=c_{3}\left[J_{0}(m)+2 \mathrm{i} J_{1}(m) \cos \left(\omega_{\mathrm{m}} t\right)\right] \exp \left(\mathrm{i} \Phi_{\mathrm{MZ}}\right)\left[u_{0}+\kappa \exp \left(\mathrm{i} \theta^{\mathrm{d}}\right) u_{1}\right] .
\end{aligned}
$$

For the longitudinal signal, we obtain the same result as in Eq. (22). The alignment signal is, however, different:

$$
W_{\mathrm{MZ}}=-4 c_{1} c_{3} J_{1}(m) \sqrt{2 / \pi} \kappa\left[\sin \left(\varphi-\Phi_{\mathrm{MZ}}-\theta^{\mathrm{d}}\right)+\sin \left(\Phi_{\mathrm{MZ}}+\theta^{\mathrm{d}}\right)\right] .
$$

At the proper operating point $\left(\varphi=\Phi_{\mathrm{MZ}}=0\right)$ this signal vanishes. We need two more modulations in order to control the phase $\Phi_{\mathrm{MZ}}$. Both $\varphi$ and $\Phi_{\mathrm{MZ}}$ are modulated sinusoidally with small modulation index at some low frequency (375 Hz and $11 \mathrm{kHz}$ in our experiment). By synchronous demodulation with the proper local oscillators, we can effectively obtain the derivatives

$$
\begin{aligned}
& \frac{\partial W_{\mathrm{MZ}}}{\partial \varphi}=-4 c_{1} c_{3} J_{1}(m) \sqrt{2 / \pi} \kappa \cos \left(\varphi-\Phi_{\mathrm{MZ}}-\theta^{\mathrm{d}}\right), \\
& \frac{\partial W_{\mathrm{MZ}}}{\partial \Phi_{\mathrm{MZ}}}=-8 c_{1} c_{3} J_{1}(m) \sqrt{2 / \pi} \kappa \sin (\varphi / 2) \sin \left(\varphi / 2-\Phi_{\mathrm{MZ}}-\theta^{\mathrm{d}}\right),
\end{aligned}
$$

and by demodulating at both modulation frequencies in series we obtain

$$
\frac{\partial^{2} W_{\mathrm{MZ}}}{\partial \Phi_{\mathrm{MZ}} \partial \varphi}=-4 c_{1} c_{3} J_{1}(m) \sqrt{2 / \pi} \kappa \sin \left(\varphi-\Phi_{\mathrm{MZ}}-\theta^{\mathrm{d}}\right) .
$$

At the proper operating point, $\varphi=\Phi_{\mathrm{MZ}}=0$, we have

$$
\begin{aligned}
& \frac{\partial W_{\mathrm{MZ}}}{\partial \varphi}=-4 c_{1} c_{3} J_{1}(m) \sqrt{2 / \pi} \kappa \cos \theta^{\mathrm{d}}, \\
& \frac{\partial W_{\mathrm{MZ}}}{\partial \Phi_{\mathrm{MZ}}}=0 \\
& \frac{\partial^{2} W_{\mathrm{MZ}}}{\partial \Phi_{\mathrm{MZ}} \partial \varphi}=4 c_{1} c_{3} J_{1}(m) \sqrt{2 / \pi} \kappa \sin \theta^{\mathrm{d}}
\end{aligned}
$$

In principle, either Eq. (33) or Eq. (35) could be used for detecting the Mach-Zehnder alignment error signals. In practice, however, Eq. (35) might be preferable, because the associated 'common mode' term (Eq. (12)) vanishes.

\subsection{Misalignments caused by individual components}

Next we have to consider what type of mode combination results from a misalignment of each individual component. For general cavities (and thus for the more complicated cavities of GEO 600 [3,16], such as a folded PR cavity or three-mirror ring-cavity modecleaners), a numerical ray-tracing code was written in Mathematica. It computes the effect of mirror misalignments on the axis of the cavity eigenmode. For simpler cavities, such as those in our prototype, the results can also be derived directly. In each case we compute the effect of the misalignment, referenced back to the beam waist and expressed as $\theta^{\mathrm{w}}$ (see Section 2.1).

$P R$ cavity. We assume a plane front mirror $\mathrm{M}_{\mathrm{PR}}$ (as in our prototype) and take as rear mirror misalignment the common mode of both end mirrors, which we now just call $\mathrm{M}_{1}$. The waist of all beams in the PR cavity is at $\mathrm{M}_{\mathrm{PR}}$.

A rotation of the end mirror $\mathrm{M}_{1}$ by an angle $\alpha_{\text {comm }}$ causes a lateral displacement of the cavity eigenmode by the distance $\Delta x=\alpha_{\text {comm }} R_{1}$, where $R_{1}$ is the radius of curvature of the end mirror. At the beam waist we thus have $\theta_{\text {comm }}^{\mathrm{w}}=0^{\circ}$.

A rotation of $\mathrm{M}_{\mathrm{PR}}$ by an angle $\alpha_{\mathrm{PR}}$ causes a lateral displacement $\Delta x=\alpha_{\mathrm{PR}}\left(R_{1}-L_{\mathrm{PR}}\right)$ at the waist, and an angular misalignment $\alpha_{\mathrm{PR}}$. Thus the cavity eigenmode, with respect to this reference, can be described as

$$
u_{0}(x, 0)+\frac{\alpha_{\mathrm{PR}}\left(R_{1}-L_{\mathrm{PR}}\right)}{w_{0}} u_{1}(x, 0)-\mathrm{i} \frac{\pi w_{0}}{\lambda} \alpha_{\mathrm{PR}} u_{1}(x, 0),
$$

and the phaseshift between $u_{1}$ and $u_{0}$ at the waist is given by

$$
\theta_{\mathrm{PR}}^{\mathrm{w}}=-\arctan \left(\frac{\pi w_{0}}{\lambda} / \frac{R_{1}-L_{\mathrm{PR}}}{w_{0}}\right)=-\arctan \frac{z_{\mathrm{R}}}{R_{1}-L_{\mathrm{PR}}} .
$$




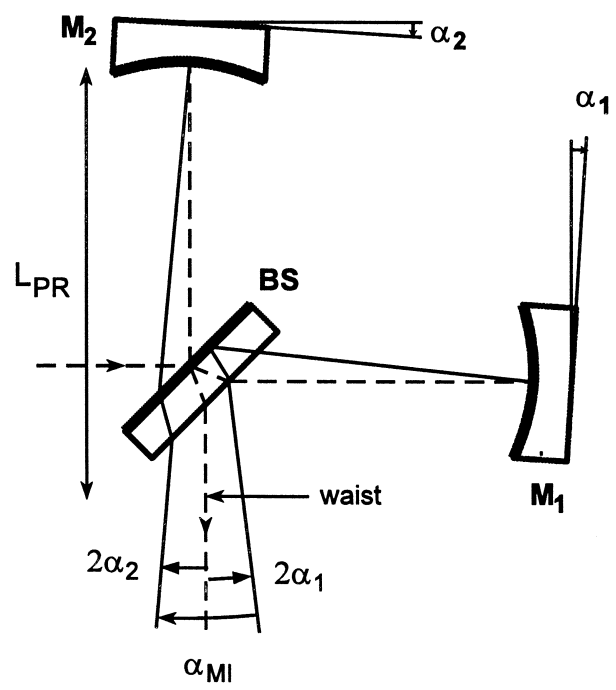

Fig. 2. A misalignment of the Michelson interferometer is described by the differential mode of the end mirrors. Due to our sign convention, $\alpha_{\text {diff }}=\alpha_{1}+\alpha_{2}$. The angle between the beams is $\alpha_{\mathrm{MI}}=2 \alpha_{\mathrm{diff}}$.

Note that the directly reflected incoming beam (our reference) experiences a rotation by $2 \alpha_{\mathrm{PR}}$. This has the additional consequence that the whole interference pattern is tilted by an angle somewhere between $\alpha_{\mathrm{PR}}$ and $2 \alpha_{\mathrm{PR}}$. This makes it necessary to employ an additional mechanism to keep the pattern centered on the quadrant detector (see Section 3.3).

Since in general both $\mathrm{M}_{\mathrm{PR}}$ and the end mirrors will be misaligned simultaneously, we will have a mixture of the two types of misalignments at the waist, described by $\theta_{\mathrm{PR}}^{\mathrm{w}}$ and $\theta_{\text {comm }}^{\mathrm{w}}$. With two separate quadrant detectors, each detecting a fraction of the beam reflected from the PR cavity, it is possible to obtain independent signals for $M_{P R}$ and the end mirrors' common mode, if an additional phase shift between $u_{0}$ and $u_{1}$ is introduced in one of the beams before detection.

For our prototype we have $R_{1}=R_{2}=33 \mathrm{~m}, L_{\mathrm{PR}}=31 \mathrm{~m}, z_{\mathrm{R}}=\sqrt{L_{\mathrm{PR}}\left(R_{1}-L_{\mathrm{PR}}\right)}=7.87 \mathrm{~m}$ and hence $\theta_{\mathrm{PR}}^{\mathrm{w}}=-75.7^{\circ}$. We placed the first quadrant detector (PD1 in Fig. 4) approximately $z=1 \mathrm{~m}$ from the waist and thus obtained $\psi=\eta(1 \mathrm{~m})$ and $\theta_{\mathrm{PR}}^{\mathrm{d}}=\theta_{\mathrm{PR}}^{\mathrm{w}}+\psi=-68.5^{\circ}$, still giving $\sin \left(68.5^{\circ}\right)=93 \%$ of the maximum possible signal for $\mathrm{M}_{\mathrm{PR}}$.

The signal for the end mirrors is obtained by the use of an adjustable lens system (L1 and L2 in Fig. 4) in front of the second detector PD2, initially designed for $\psi=90^{\circ}$ phase shift, but adjusted such that $\theta_{\mathrm{PR}}^{\mathrm{d}}=\theta_{\mathrm{PR}}^{\mathrm{w}}+\psi=0^{\circ}$ at PD2, corresponding to $\theta_{\mathrm{comm}}^{\mathrm{d}}=75.7^{\circ}$ and $97 \%$ of the maximum possible signal. The lens system was adjusted experimentally by dithering the orientation of $\mathrm{M}_{\mathrm{PR}}$ and adjusting for vanishing signal at the dithering frequency in PD2's output signal. It was not necessary to further separate the error signals. The design of the lens system is briefly described in Section 2.4 .

Michelson interferometer. Again we describe the misalignments of the Michelson at the beam waist, which is at a distance $L_{\mathrm{PR}}$ from the end mirrors. With external modulation the local oscillator beam was taken from the first arm as reflection from the back of the beamsplitter, which has a finite reflectivity of $290 \mathrm{ppm}$. Thus the beam returning from the first arm acted as angular reference. According to Fig. 2, we have at the waist an angle $\alpha_{\mathrm{MI}}=2 \alpha_{\text {diff }}=2\left(\alpha_{1}+\alpha_{2}\right)$ and a lateral displacement $\Delta x=-\alpha_{\mathrm{MI}} L_{\mathrm{PR}}$. Similar to the derivation of Eq. (37), we find for the phase between $u_{1}$ and $u_{0}$

$$
\theta_{\mathrm{MI}}^{\mathrm{w}}=\arctan \left(-\frac{z_{\mathrm{R}}}{L_{\mathrm{PR}}}\right),
$$

which amounts to $-14.25^{\circ}$ in our prototype. Since the best signal is again obtained with $\theta_{\mathrm{MI}}^{\mathrm{d}}=90^{\circ}$ at the detector (see Eq. (23)), we need another lens system (L3 and L4 in Fig. 4) to introduce approximately $104.25^{\circ}$ of extra phase shift between $u_{1}$ and $u_{0}$.

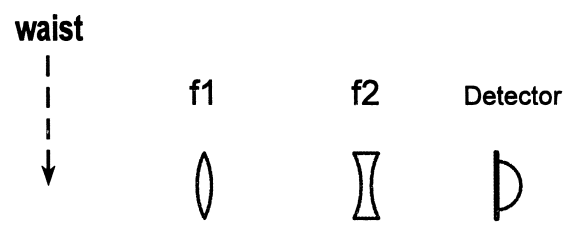

Fig. 3. Scheme of the lens system used to introduce additional Guoy phase shift between $u_{1}$ and $u_{0}$. 
Table 1

Parameters of the two lens systems used in our prototype (see also Fig. 3). The beam diameters $2 w$ are given at the first and second lens, and at the detector

\begin{tabular}{cccccccccc}
\hline & $\begin{array}{c}d_{1} \\
{[\mathrm{~cm}]}\end{array}$ & $\begin{array}{c}2 w_{1} \\
{[\mathrm{~mm}]}\end{array}$ & $\begin{array}{c}f_{1} \\
{[\mathrm{~cm}]}\end{array}$ & $\begin{array}{c}d_{2} \\
{[\mathrm{~cm}]}\end{array}$ & $\begin{array}{c}2 w_{2} \\
{[\mathrm{~mm}]}\end{array}$ & $\begin{array}{c}f_{2} \\
{[\mathrm{~cm}]}\end{array}$ & $\begin{array}{c}d_{3} \\
{[\mathrm{~cm}]}\end{array}$ & $\begin{array}{c}2 w_{\mathrm{D}} \\
{[\mathrm{mm}]}\end{array}$ & $\begin{array}{c}\psi_{\text {total }} \\
{[\mathrm{deg}]}\end{array}$ \\
\hline PR cavity & 120 & 2.3 & 35 & 32.17 & 0.22 & -3 & 50 & 1.78 & 90 \\
Michelson & 50 & 2.27 & 25 & 23.27 & 0.175 & -2 & 55 & 2.10 & 104 \\
\hline
\end{tabular}

With Schnupp modulation, the "local oscillator" consists of the phase modulation sidebands that leak out at the dark fringe port because of the armlength difference. Their geometry is determined by the eigenmode of the PR cavity, which we assume to be well aligned by its own autoalignment system. The ratio of lateral displacement to angular misalignment and hence $\theta_{\mathrm{MI}}^{\mathrm{w}}$ remain the same.

Mach-Zehnder alignment We assume that both beamsplitter and RP are made such that their two surfaces are parallel to each other and that their material and shape are identical. It can be shown that under these conditions a tilt of RP will cause a pure lateral displacement between the two beam axes (of the dark fringe beam and the local oscillator beam). In our prototype we did not install any automatic alignment system for RP. If we were rotating RP we would simultaneously change the phase shift between the two interfering beams. This phase shift needs to be well controlled to keep the interferometer in lock. The dynamic range of the only other control element for this phase shift, Pockels cell PC3, was too small to permit feedback of alignment correction signals to RP without throwing the interferometer out of lock.

\subsection{Computation of lens systems}

We now describe a lens system for the purposes mentioned above, which ideally fulfills the following requirements: arbitrary design value of phase shift $\psi$ (usually around $90^{\circ}$ ), preferably adjustable around the design value, compactness and reasonable spot size on the quadrant detector.

We computed such lens systems using the matrix formalism (see, e.g., Ref. [17]). One starts at the beam waist and traces the beam parameter $q$ through sections of space and lenses. Care must be taken in the computation of the Guoy shift. We are not interested in an absolute value of the Guoy shift, but only in the difference between the $u_{0}$ and $u_{1}$ modes. This difference must be continuous, in particular at thin lenses. Thus it is not possible simply to multiply the matrices and use only the product.

With a Mathematica program we computed the phase shift and beam size in various lens systems and found reasonable solutions for the two cases of interest in our experiment, which require a phase shift of $90^{\circ}$ and $104^{\circ}$, respectively. Each lens system consists of one converging lens (focal length $\left.f_{1}\right)$ and one diverging lens $\left(f_{2}\right)$, separated by a distance $d_{2} \approx f_{1}+f_{2}$ (see Fig. 3 and Table 1). The beam radius at the detector was designed to be approximately $2 \mathrm{~mm}$. The lens systems can be adjusted via the distance $d_{2}$ between the two lenses. It turns out that very close to the desired position (which yields the design phase shift) the beam radius at the detector has a minimum, which simplifies the initial setup. By moving the second lens $1 \mathrm{~cm}$ in either direction, the phase shift $\psi$ can be varied by more than $\pm 30^{\circ}$, thus allowing final adjustment of the phase shift.

\section{Experimental setup}

\subsection{Overview and longitudinal loops}

The experiments were carried out in 1996-97 at the Garching $30 \mathrm{~m}$ prototype interferometer, which has recently been described elsewhere [8]. Here we discuss only the loops that are relevant for the autoalignment system (see Fig. 4), and for brevity only the setup using external modulation. After changing to Schnupp modulation in late 1997, all autoalignment systems continued to work without modification, even after the recent implementation of dual recycling [19].

The light source is an argon ion laser running at $514 \mathrm{~nm}$. Its light is brought into the vacuum chamber with a single-mode fiber, at the end of which we get up to $300 \mathrm{~mW}$ of single mode light. The beam injector unit which holds the fiber output coupler is suspended as a pendulum of approximate length $0.7 \mathrm{~m}$, as are the beamsplitter $\mathrm{BS}$, end mirrors $\mathbf{M}_{1}$ and $\mathbf{M}_{2}$, Pockels cell PC3 and recombination plate RP. Only BS and RP are suspended as double pendulums, all others as single pendulums. 


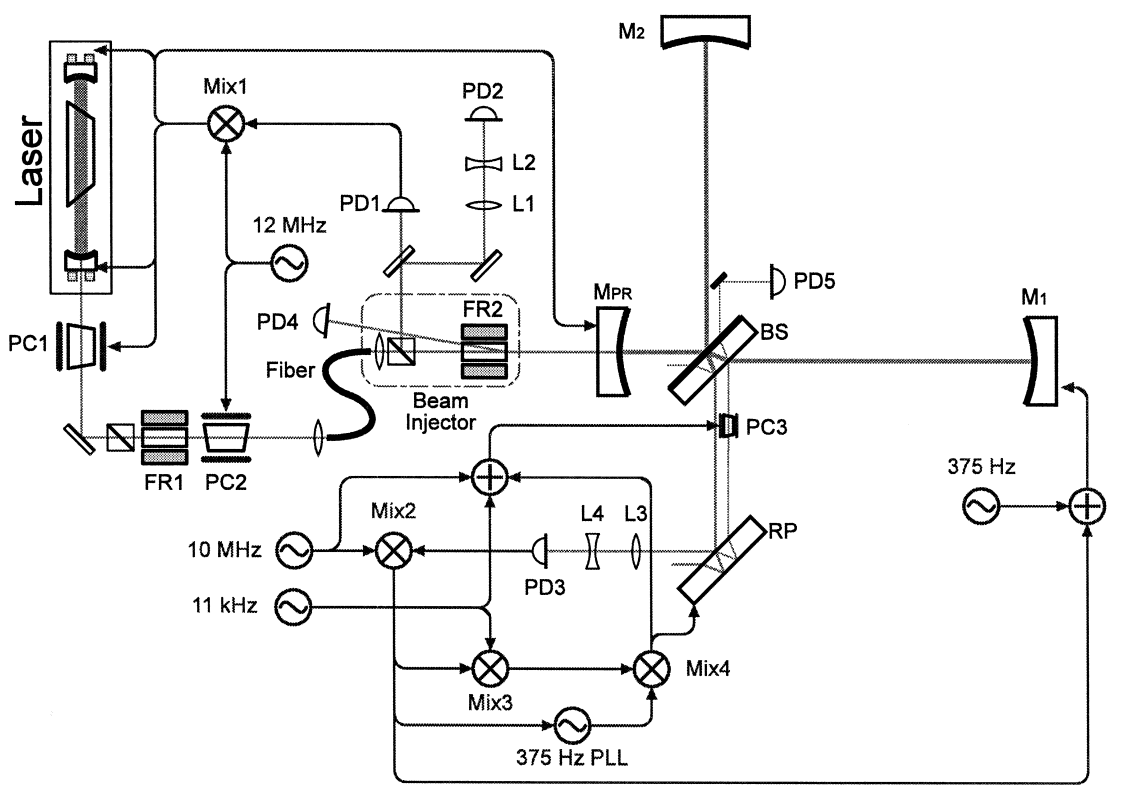

Fig. 4. Simplified diagram of the $30 \mathrm{~m}$ prototype experiment. Only the longitudinal control loops for the PR cavity lock, Michelson dark fringe lock and Mach-Zehnder phase lock are shown. This diagram shows the external modulation setup, which was later changed to Schnupp modulation.

The laser frequency is locked to the PR cavity using the Pound-Drever scheme. The $12 \mathrm{MHz}$ phase modulation is applied by Pockels cell PC2 with a modulation index of approximately 0.2 rad. The light reflected from the PR mirror (or cavity) is separated by a Faraday isolator on the beam injector unit, directed out of the vacuum chamber and detected by the quadrant detectors PD1 and PD2 (described below). The longitudinal Pound-Drever loop uses the $12 \mathrm{MHz}$ sum output of PD1 to generate the error signal and has a bandwidth of several $100 \mathrm{kHz}$.

The Michelson phase is detected with external modulation. Around $290 \mathrm{ppm}$ of the light returning from $\mathrm{M}_{1}$ is taken as reference beam. It is phase modulated at $10 \mathrm{MHz}$ by the suspended Pockels cell PC3, with a modulation index of approximately $0.25 \mathrm{rad}$. The dark fringe beam and the reference beam interfere at the central part of the recombination plate $\mathrm{RP}$, where it has a 50:50 coating. This arrangement is known as Mach-Zehnder interferometer. PD3 detects the output light. Its $10 \mathrm{MHz}$ sum output is used for the dark-fringe lock, via feedback to both end mirrors, with a unity-gain frequency (UGF) of up to several $\mathrm{kHz}$.

In the external modulation setup, further loops are necessary in order to keep the Mach-Zehnder phase locked. They make use of the $375 \mathrm{~Hz}$ and $11 \mathrm{kHz}$ signals shown in Fig. 4. Their complexity was one of the reasons for switching to Schnupp modulation instead.

\subsection{Quadrant photodetectors}

All three quadrant photodetectors PD1, PD2 and PD3 are built similarly. We use Centronics QD50-0 photodiodes in the photoconductive mode with a reverse bias voltage of $15 \mathrm{~V}$. The current from each quadrant is processed by a separate front end having two output signals each: voltages proportional to the DC photocurrent (bandwidth $400 \mathrm{kHz}$ ) and the RF signal at 10 or $12 \mathrm{MHz}$. For the RF channel we have a noise-equivalent photocurrent of approximately $35 \mu \mathrm{A}$ per quadrant, which is dominated by the Johnson noise in the real part of the source impedance $(2 \mathrm{k} \Omega)$.

These DC and RF signals are then separately processed with analog electronics to produce a total of six output signals from each quadrant detector. The RF signals $V$ and $W$ are the ones defined in Eqs. (12) and (13), and the DC signals $\bar{V}$ and $\bar{W}$ are their DC counterparts, formed by combining the DC outputs of the quadrants. $X$ and $Y$ represent the two orthogonal directions on the surface of the quadrant photodiode.

$V$ : This signal is used for the longitudinal loops (PR cavity Pound-Drever loop and Michelson dark-fringe loop, respectively).

$W_{X}, W_{Y}$ : These are the most important output signals for the autoalignment system, describing the tilt of the wavefronts of the interfering beams against each other. They are demodulated in the detector and have a bandwidth of about 70 $\mathrm{kHz}$. 
$\bar{V}: \quad$ This signal (total DC photo current) is used for monitoring and normalization purposes.

$\bar{W}_{X}, \bar{W}_{Y}$ : These two signals (differences of the DC photo currents) are fed to the beam-steering mechanism described in Section 3.3 below and are kept near zero by that beam-steerer.

\subsection{Auxiliary beam-steering loops}

As we mentioned in Section 2.2, the quadrant detectors can produce reliable alignment error signals only when the interference pattern is centered on the photodiode. Since in the course of the alignment (either manual or automatic) the position and angle of the beams leaving the vacuum chamber are changed (e.g. by rotating $\mathrm{M}_{\mathrm{PR}}$ ), we need an extra loop that centers the beam on the quadrant diode for each of the detectors PD1, PD2 and PD3. These loops make use of the DC output signals $\bar{W}_{X}$ and $\bar{W}_{Y}$ from the quadrant detectors. To deflect the beam we use commercial magnet-mirror units modified for our purpose. The UGF of these loops is around 30-50 Hz, sufficient for our purposes.

A modification was necessary in the loop for PD3, because here the DC light level hitting the photodetector may vary widely during lock acquisition. Thus the $\bar{W}_{X}$ and $\bar{W}_{Y}$ signals from PD3 are divided by the DC sum signal $\bar{V}$ from the same detector before they are used as error signals. For GEO 600 we will use two commercial galvanometer scanners mounted orthogonally for each detector. In a prototype we have obtained a UGF of $2 \mathrm{kHz}$ with these units. It is important for these beam-steering loops to have a bandwidth considerably higher than the main autoalignment loop of which they are a part.

\section{Automatic alignment loops}

\subsection{Actuators}

The orientation of all suspended components can be controlled by three magnets attached to each pendulum, with coils nearby. Together with shadow sensors built into the coil holders, these coil-magnet systems are used for the "local control", systems that actively damp the pendulum resonances (see e.g. Ref. [18] and references therein). We use them simultaneously to control the position and orientation of the suspended components.

The transfer functions from the respective control inputs (translation, rotation, tilt) to an actual component movement involve the mechanics of the pendulums and the action of the local controls. Fortunately, all such transfer functions in our prototype have a similar simple shape, which can be approximated by two-pole lowpass models

$$
H_{\mathrm{P}}(s=\mathrm{i} \omega)=\frac{A_{\mathrm{P}}}{1+s / \omega_{\mathrm{P}} Q_{\mathrm{P}}+s^{2} / \omega_{\mathrm{P}}^{2}} .
$$

Their pole frequencies $f_{\mathrm{P}}=\omega_{\mathrm{P}} /(2 \pi)$ are between 0.5 and $2 \mathrm{~Hz}$, and the $Q_{\mathrm{P}}$ of these resonances is quite low, i.e. between 0.7 and 4, due to the active damping by the local control modules. For each channel, Table 2 shows these parameters, together with the overall factors $A_{\mathrm{P}}$ and the sensor gains $A_{\mathrm{S}}$.

\subsection{Loop filters}

We have tried several variants of loop filters. The purpose of all of them is to allow the UGF to be around or above the pendulum resonance and to provide extra gain at low frequencies (below the resonance). Because the overall loop gain may

Table 2

Calibration parameters of the auto-alignment loops. 'EM diff.' is the differential mode of the end mirrors (Michelson alignment), 'EM comm.' their common mode (PR cavity alignment), and PR refers to the power recycling mirror. $A_{\mathrm{P}}, f_{\mathrm{P}}$ and $Q_{\mathrm{P}}$ describe the pendulum response (Eq. (39)). The sensor gain $A_{\mathrm{S}}$ includes everything from a mirror motion to a signal at the photodetector's output

\begin{tabular}{lcclc}
\hline Mode & $\begin{array}{c}A_{\mathrm{P}} \\
{[\mu \mathrm{rad} / \mathrm{V}]}\end{array}$ & $\begin{array}{c}f_{\mathrm{P}} \\
{[\mathrm{Hz}]}\end{array}$ & $Q_{\mathrm{P}}$ & $\begin{array}{c}A_{\mathrm{S}} \\
{[\mathrm{V} / \mathrm{rad}]}\end{array}$ \\
\hline EM diff. X & 28.7 & 0.9 & 3.26 & $3.0 \times 10^{6}$ \\
EM diff. Y & 20.8 & 0.9 & 3.0 & $2.6 \times 10^{6}$ \\
EM comm. X & 287 & 0.89 & 2.99 & $58 \times 10^{3}$ \\
EM comm. Y & 208 & 0.9 & 2.63 & $47 \times 10^{3}$ \\
PR X & 2500 & 1.84 & 2.6 & $23 \times 10^{3}$ \\
PR Y & 3500 & 1.67 & 1.99 & $25 \times 10^{3}$ \\
\hline
\end{tabular}


change according to many parameters, we have tried to normalize the error signals as far as possible by analog division (see Section 4.3) and also to use unconditionally stable loops wherever possible.

The favorite design consists of a ' $f^{1 / 2}$ filter' active at frequencies above the pendulum resonance. Such a filter can be built by cascading poles and zeroes of the transfer function separated by half a decade in frequency. The result is an open-loop gain curve that rolls off approximately with $f^{-3 / 2}$ above the pendulum resonance and has a phase margin of around $45^{\circ}$ (assuming there are no extra phase delays). At frequencies below the pendulum resonance, there is an integrator active to increase the gain at low frequencies and DC.

An alternative design employs a biquadratic active filter to compensate the pendulum resonance. A new pole is introduced at a frequency above the loop's UGF, e.g. at $20 \mathrm{~Hz}$. It is thus possible to obtain an open-loop gain that continuously rolls off as $f^{-1}$ (or $f^{-3 / 2}$ if desired) even around the pendulum resonance frequency, at the cost of increased circuit complexity.

During the development of our system, we have built both kinds of filter, and both are still used in the prototype. For new systems we would prefer the first kind, because it is easier to build (no exact knowledge of the pendulum resonance is necessary) and because the biquadratic filter of the alternative design intrinsically has very small dynamic range.

\subsection{Auxiliary signals for normalization}

The two single-element photodetectors PD4 and PD5 in Fig. 4 are used to normalize the autoalignment error signals. PD4 detects a stray beam from the beam injector, which is a sample of the injected light hitting $\mathrm{M}_{\mathrm{PR}}$. PD5 detects a sample of the light power circulating in the PR cavity, which depends on the alignment of the interferometer, the condition of beamsplitters and mirrors, and in particular on the locking state of the longitudinal loops.

The error signals for the Michelson interferometer (from PD3) are proportional to the product of the amplitudes of the beam returning from one arm and of the reference beam. Both of these amplitudes are proportional to the amplitude of the light circulating in the PR cavity, and hence the error signal is proportional to the power of that light. Therefore, the error signals are divided by the output from photodetector PD5 with an analog divider before being fed to the loop filters.

The error signals for the PR cavity are, however, proportional to the product of the amplitudes of the beam injected into the interferometer and of the beam circulating in the PR cavity. The power of those two beams is measured with photodetectors PD4 and PD5, respectively. Therefore we compute the square root of the product of these powers with an analog circuit and use it as denominator to normalize all four PR cavity error signals from PD1 and PD2.

\subsection{Alignment loops for Michelson and PR cavity}

The system used for aligning the Michelson interferometer is the most important automatic alignment loop in our setup, because any noticeable Michelson misalignment immediately reduces the stability of the longitudinal loops. As described in the previous sections, the two error signals are obtained from the quadrant detector PD3. The loop filters were built according to the first design of Section 4.2, with an $f^{1 / 2}$ filter active from $1.5 \mathrm{~Hz}$ to $45 \mathrm{~Hz}$, an integrator between $3 \mathrm{mHz}$ and $300 \mathrm{mHz}$ and an additional two-pole lowpass filter at $85 \mathrm{~Hz}$ in each channel. The UGF is around $10 \mathrm{~Hz}$ in normal operation and can be increased up to around $20 \mathrm{~Hz}$ if desired.

Error signals that describe the misalignment of the PR cavity are obtained from the quadrant detectors PD1 (for the PR mirror) and PD2 (for the end mirrors' common mode alignment). The loop filters for the PR mirror were built according to the second design of Section 4.2 above with the new pole introduced at $20 \mathrm{~Hz}\left(Q_{\text {pole }}=1.5\right)$, an $f^{-1 / 2}$ filter active between $80 \mathrm{mHz}$ and $2.5 \mathrm{~Hz}$, and an integrator starting at $5 \mathrm{mHz}$ in each channel. The UGF is near $5 \mathrm{~Hz}$ in normal operation and can be increased up to about $10 \mathrm{~Hz}$.

The Michelson loop and the PR cavity loop both act on the end mirrors, and the separation of 'common mode' and 'differential' signals is not perfect. Therefore there is some interaction between these loops. In particular we found that the Michelson loop should have a higher UGF than the two channels of the PR cavity loop that act on the end mirrors, and also that the Michelson loop should be switched on first. Otherwise, if one tries to automatically align the PR cavity with no or too little gain of the Michelson loop, a common mode signal fed back to the end mirrors invariably involves some differential mode component, which reduces the Michelson contrast or may even throw one of the longitudinal loops (and hence the whole interferometer) out of lock.

\subsection{Spot positions on end mirrors}

The loops that control the positions of the spots on the end mirrors are much less critical than those described above. The error signals are obtained from position sensitive detector (PSD) diodes mounted behind the end mirrors $\mathrm{M}_{1}$ and $\mathrm{M}_{2}$. The control signals are fed back to the beam injector and beamsplitter, respectively. The open loop gain of these loops has a simple $1 / f$ dependence, with a UGF of around $0.3 \mathrm{~Hz}$. 
The PSD diodes can be positioned behind the end mirrors with motorized mounts. When everything is in lock, the spot positions on either of the end mirrors can thus be controlled by moving the PSD with the motors, while all other spot positions remain fixed and the Michelson and PR cavity are kept well aligned. The beam position on the end mirror can thus be fixed to any desired spot, such as the center of the mirror (to minimize torsional coupling) or to the spot that yields the best Michelson contrast.

\subsection{Lock acquisition and error checking}

The normalization of all error signals described in Section 4.3 involves analog division by a voltage that is proportional to some light level inside the interferometer. If any part of the system falls out of lock, most of these light levels quickly drop to very low levels. The normalized signal then gets very inaccurate or even saturated. To prevent feeding back such "wrong", error signals to the suspended components, the denominators used for normalization are continually monitored by analog comparators. Once a denominator drops below a preset threshold, an analog sample-and-hold circuit is activated that holds the correction signal (output of the loop filter) at its last value. For initial manual alignment, there is another mode of operation which forces all correction signals to zero.

In practice these error checking systems worked sufficiently well if the error that led to the drop in light power was not caused by the autoalignment system itself. If, however, something is wrong with one of the autoalignment loops, then usually one or several components start to move away from their aligned positions, until the interferometer falls out of lock. The sample-and-hold systems then hold the components in positions that are already removed quite far from the optimum alignment, and lock acquisition has to be restarted from the initial positions of all components.

Thus we monitor the correction signals during operation, and manually adjust offsets from time to time such that the correction signals applied by the autoalignment loops are near zero. Then, in case of a lock reacquisition, the 'zero' position for all component orientations is already very close to the optimum position. In the planned large detectors, this task would be performed by a computerized control system, which is also responsible for monitoring the locking status, lock acquisition, etc. With such a system available, we would no longer use the sample-and-hold circuits but instead switch the correction signal to zero in case of insufficient light levels.

One important conclusion that we draw from our experiments is that this autoalignment system can only start to work after all longitudinal loops of the interferometer are already locked. Thus the interferometer must be started up with manual alignment, until the PR cavity and Michelson dark fringe are both locked. In particular care must be taken that the PR cavity locks in its fundamental $\mathrm{TEM}_{00}$ mode. Only then can the autoalignment system be activated.

\subsection{Noise spectra}

We have measured the noise spectra of all loops at their error points both with the loops switched on and off. They were divided by the sensor transfer function $H_{\mathrm{S}}(s)$ to give the mirror motion in radians. For verification we have also measured the spectra of the correction signals applied to the mirrors when the loop was working. These were multiplied with the pendulum transfer function $H_{\mathrm{P}}(s)$, such that they also express the mirror motion. A typical set of such measurements is shown in Fig. 5.

The upper two curves show the error signal with the loop switched off and the correction signal with the loop in operation. The solid curve shows the error signal with the loop on. The lowest curve shows the sensor noise, measured with

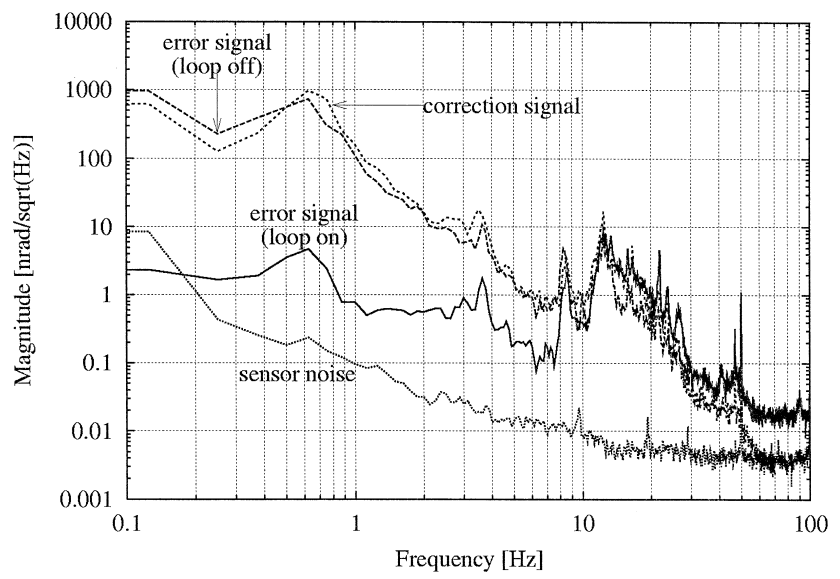

Fig. 5. Noise spectrum of the $x$-channel of the Michelson alignment loop, calibrated in $\mathrm{rad} / \sqrt{\mathrm{Hz}}$ of differential end mirror motion (see text). 
Table 3

Integrated rms alignment noise from $0.1 \mathrm{~Hz}$ to $100 \mathrm{~Hz}$. 'EM diff.' is the differential mode of the end mirrors (Michelson alignment), 'EM comm.' their common mode (PR cavity alignment), and PR refers to the power recycling mirror

\begin{tabular}{lccc}
\hline Mode & $\begin{array}{l}\text { Loop } \\
\text { off }\end{array}$ & $\begin{array}{l}\text { Loop on } \\
{\left[\mathrm{nrad}_{\mathrm{rms}}\right]}\end{array}$ & $\begin{array}{l}\text { Corr. } \\
\text { signal }\end{array}$ \\
\hline EM diff. X & 461 & 11 & 522 \\
EM diff. Y & 1100 & 26 & 790 \\
EM comm. X & 1390 & 26 & 1540 \\
EM comm. Y & 234 & 34 & 1010 \\
PR X & 1300 & 270 & 1000 \\
PR Y & 2700 & 176 & 2730 \\
\hline
\end{tabular}

the same average photocurrent as that appearing during operation, produced by white light from an incandescent lamp. We have numerically integrated the noise spectra between $0.1 \mathrm{~Hz}$ and $100 \mathrm{~Hz}$ to obtain rms values for the angular misalignments (see Table 3).

Since some of the curves were measured on different days, and the environmental motion of our lab depends on outer conditions, there is considerable variability in the data, especially in the measurements with the loop off and those of the correction signal (which both represent the amount of alignment noise without the loop). These rms values are usually dominated by a DC term, which also depends on how well the interferometer was manually aligned. The real usefulness of the autoalignment system is only incompletely described by these numbers, since the natural fluctuations increase even more towards frequencies below $0.1 \mathrm{~Hz}$. These slow drifts are well compensated by the loops, but not easily measured.

The remaining fluctuations with the loop in operation are somewhat more reproducible. For the Michelson alignment, we find an rms deviation of 11 and $26 \mathrm{nrad}$ for the $X$ and $Y$ channels, respectively. That corresponds at the waist (near the beamsplitter) to an rms lateral displacement between the axes of the two beams of $0.33 \mu \mathrm{m}$ and $0.78 \mu \mathrm{m}$, respectively, or equivalently to a fraction of $2.8 \times 10^{-4}$ and $6.7 \times 10^{-4}$ of the beam radius $w_{0}$.

For the four other channels (those for the PR cavity) the results look qualitatively similar. In these channels, however, the sensor noise was higher by a factor of around 100. It was later found that during these measurements a spurious Fabry-Perot had been formed between the ends of the fiber that brings the light into the tank. The resulting excess noise on the light entering the interferometer had dominated the error signals.

Another question to be addressed is that of extra noise being fed into the interferometer by the autoalignment loops. This is indeed a nontrivial problem since the loops need to act on the end mirrors. In our prototype, we used simple $1 / f^{2}$ filtering with corner frequencies of $20 \mathrm{~Hz}$ or $85 \mathrm{~Hz}$, as described in Section 4.4. Additionally there is the $1 / f^{2}$ filtering action of the pendulum itself (see Section 4.1). For the large-scale interferometers, more elaborate filtering may be necessary in order to prevent feeding noise to the mirrors at measurement frequencies. This will depend on the necessary loop bandwidths of the alignments loops, which in turn depend on how much alignment noise there is to be controlled. In our prototype, the coil-magnet actuators directly acted on the test masses, whereas in GEO 600 they will act on an intermediate mass of a double or triple pendulum, which provides additional isolation, but also a more complicated transfer function.

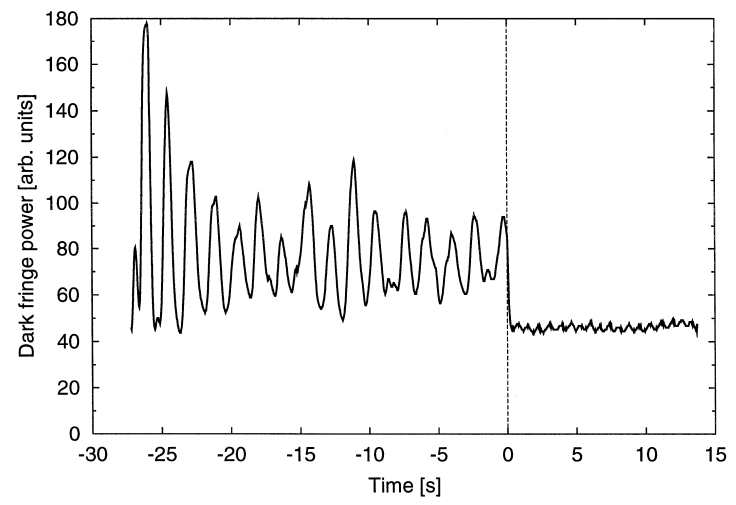

Fig. 6. Power leaving the dark fringe port of the interferometer (measured with PD3) without and with auto-alignment (switched on at $t=0$ ). The final level of 50 units corresponds to about $0.1-0.2 \%$ of the power at the beamsplitter. 


\subsection{Dark fringe contrast}

Fig. 6 shows how the light power at a dark fringe fluctuates without the auto-alignment system, and how the fluctuations are reduced after the alignment system was switched on. These environmental fluctuations shown are typical for a short time after the interferometer was manually well aligned. Actually it is very difficult to align the interferometer manually, in particular the end mirrors' common mode. In practice the fine alignment of the interferometer was done using the auto-alignment system, which was then switched off to demonstrate the natural fluctuations. On longer timescales (several minutes or longer) the contrast without auto-alignment usually deteriorates even more than shown in Fig. 6 due to slow alignment drifts.

\section{Conclusion}

The automatic beam alignment system described in this paper was the first one to completely control the alignment of a suspended Michelson interferometer with power recycling. It is now routinely used in the Garching $30 \mathrm{~m}$ prototype, and several recent experiments (high PR gain, dual recycling) would not have been possible without it. In particular the angular common mode misalignment of the end mirrors is practically impossible to align without this system, and the stability of operation is very much improved. It is also the basis for the automatic beam alignment system for GEO 600 which is under construction.

\section{Acknowledgements}

This work would not have been possible without the excellent work done by our technicians and engineers H. Klein, H. Emme and A. Weidner, who built most of the specialized hardware. KAS would like to acknowledge support from PPARC and the University of Glasgow.

\section{References}

[1] A. Abramovici et al., Science 256 (1992) 325.

[2] C. Bradaschia et al., Nucl. Instr. Meth. Phys. Res. A 298 (1990) 518.

[3] K. Danzmann et al., in: Proc. First Edoardo Amaldi Conf. on Gravitational Wave Experiments, Frascati 1994, World Scientific, Singapore, 1995, pp. 100-111.

[4] K. Tsubono et al., in: Proc. First Edoardo Amaldi Conf. on Gravitational Wave Experiments, Frascati 1994, World Scientific, Singapore, 1995, pp. 112-114.

[5] L. Schnupp, Talk at the European Collaboration Meeting on Interferometric Detection of Gravitational Waves, S. Agnello/Sorrento, Italy, 1988.

[6] C.N. Man et al., Phys. Lett. A 148 (1990) 8.

[7] R.W.P. Drever et al., in: P. Meystre, M.O. Scully (Eds.), Quantum Optics, Experimental Gravitation, and Measurement Theory, Plenum Press, New York, 1983, pp. 503-514.

[8] D. Schnier et al., Phys. Lett. A 225 (1997) 210.

[9] E. Morrison, B.J. Meers, D.I. Robertson, H. Ward, Appl. Optics 33 (1994) 5041.

[10] E. Morrison, B.J. Meers, D.I. Robertson, H. Ward, Appl. Optics 33 (1994) 5037.

[11] A. Rüdiger et al., Optica Acta 28 (1981) 641.

[12] Y. Hefetz, N. Mavalvala, D. Sigg, J. Opt. Soc. Am. B 14 (1997) 1597.

[13] D.Z. Anderson, Appl. Optics 23 (1984) 2944.

[14] R.W.P. Drever et al., Appl. Phys. B 31 (1983) 97.

[15] P. Nelson, private communication.

[16] H. Lück, Class. Quantum Grav. 14 (1997) 1471.

[17] A. Yariv, Quantum Electronics, 3rd ed., Wiley, New York, 1989.

[18] J. Winterflood, D.G. Blair, R. Schilling, M. Notcutt, Rev. Sci. Instrum. 66 (1995) 2763.

[19] G. Heinzel et al., Experimental Demonstration of a Suspended Dual Recycling Interferometer, to appear in Phys. Rev. Lett. (scheduled for 12 December 1998). 